\title{
Postoperative Transient Intractable Hyperacusis in Posterior Cingulate Gyrus Lesion Causing Partial Complex Seizure
}

\author{
FHCHOWDHURY ${ }^{\mathrm{a}}$, MR HAQUE ${ }^{\mathrm{b}}$
}

\begin{abstract}
Summary:
A young man of 19 who underwent surgical excision of a small right posterior cingulate gyrus cystic lesion for complex partial seizure. Postoperatively he developed intractable hyperacusis and persisted for several days. Here we report the case of post operative intractable hyperacusis that developed after surgery in posterior cingulate gyrus and in the literature
\end{abstract}

\section{Introduction:}

Isolated lesion in cingulated gyrus causing partial seizure is not uncommon. Cingulate gyrus is an important part of limbic system that deals with memory processing and emotional aspects of sensory input to brain. There are some connections and association of cingulate gyrus with hearing mechanism. Here we report transient intractable hyperacusis after surgical excision of a small lesion in right sided posterior cingulated gyrus through inter hemispheric approach.

\section{Case report:}

A right handed young university student of 19 years presented with complex partial seizure for 5 years. There was dizziness followed by paresthesias in left foot followed by unconsciousness (and fall) for 80-100 sec. There was no history of olfactory or auditory hallucination, tonic-clonic events or bowel-bladder incontinence. He had no history hearing disturbances, tinnitus or vertigo. Initially there was 1-3 attack of such event per year that began to increase in frequency. The frequency was 2-3 attack per month in the last year before surgery. From the beginning he was on

1. Dr. Forhad Hossain Chowdhury, Assistant professor, Neurosurgery, National Institute of Neurosciences and Hospital, Dhaka,

2. Dr. Md. Raziul Haque, Associate professor, Dept. of Neurosurgery, Dhaka Medical college Hospital, Dhaka.

Address Correspondence to: Chowdhury Forhad Hossain, Assistant professor, Neurosurgery, National Institute of Neurosciences and Hospital, Dhaka, Bangladesh. Phone +8801711949570, e-mail- forhadchowdhury74@yahoo.com

Received: 28 July 2015

Accepted: 6 October 2016 probably this is the first report of hyperacusis related to posterior cingulate gyrus surgery.

Key Words: Posterior cingulate gyrus lesion, Complex partial seizure, Hyperacusis.

(J Bangladesh Coll Phys Surg 2016; 34: 225-227)

antiepileptic (pre operatively he was on tab. carbamazepine $200 \mathrm{mg}$ two tab. 8 hourly and tab phenytoin $100 \mathrm{mg}$ one tab. 8hourly).His hearing tests including Pure Tone Audiometry (PTA) were normal. Interictal Scalp EEG reported abnormal electrical discharge from right medial parietal cortex. MRI showed a small $(1 \times 1.5 \times 1.5 \mathrm{~cm})$ cystic lesion on posterior cingulate gyrus on right side (Fig. 1A,B\&C). On MRI middle ear and internal ear anatomy was noirmal on both sides. The lesion was initially static for four years. Last followup MRI showed slight increase in size of the lesion. Then after counseling (regarding seizure and nature of lesion) we went for surgical excision. The operation was performed under general anesthesia (Anaesthesia was induced with thiopentone $300 \mathrm{mg}$ and fentanyl $125 \mathrm{ig}$. Tracheal intubation was facilitated with suxamethonium $90 \mathrm{mg}$. Anaesthesia was maintained with halothane in a mixture of $\mathrm{O}_{2}$ and $\mathrm{N}_{2} \mathrm{O}$ in addition to intermittent doses of vicuronium $(0.1 \mathrm{mg} / \mathrm{kg})$ and fentanyl. Monitoring parameters included were ECG, pulse oximetry , endtidal capnography, arterial BP, central venous pressure (CVP), and urine output. ) We removed the lesion through interhemispheric approach after posterior parietal craniotomy extending on the both side of sagital suture with right sided preponderance.Post operatively he was on inj.Phenytoin, inj ceftiraxone, inj.omeprazole and diclofenac suppository. From $2^{\text {nd }}$ post operative day (POD) patient developed severe intractable hyperacusis. For which he had to isolate in a silent room where we communicated with very few words in very slow volume. Even then at that time he used to put a cotton ball in both external acoustic canals. This hyperacusis persisted in same intensity for next five 


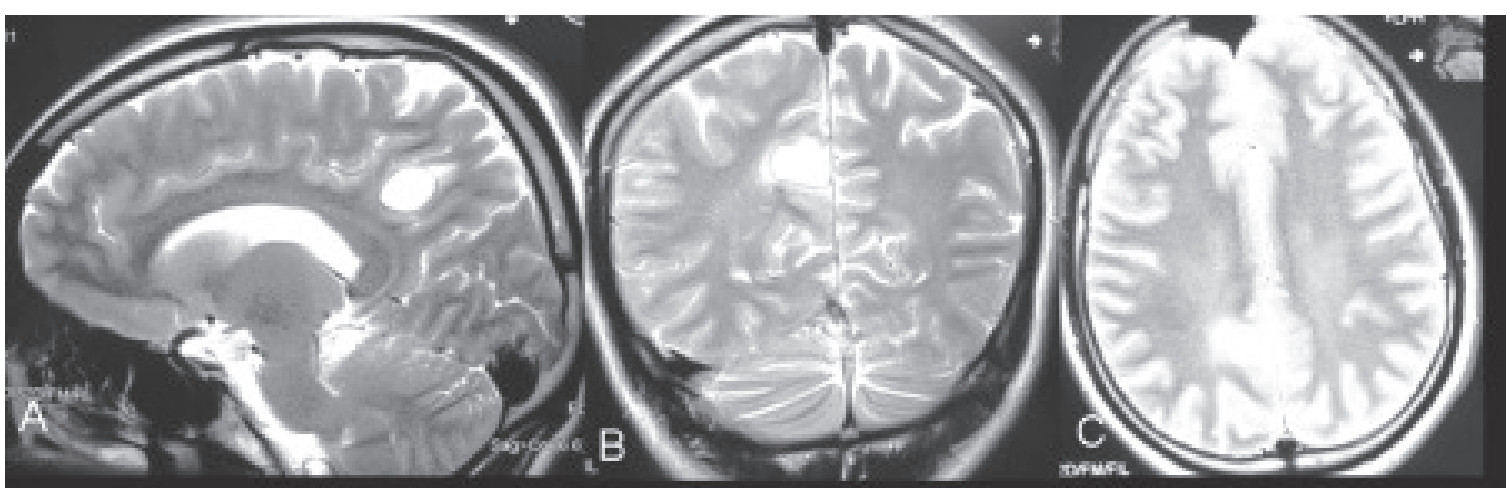

Fig.-1: MRI of brain T2W saggital (A), coronal (B) and axial (C) images showing posterior cingulate gyrus cystic Lesion.

days. From 8th POD the intensity of hyperacusis began to decrease and it became tolerable on $12^{\text {th }}$ POD and hearing became normal on $21^{\text {st }}$ POD. After operation he was seizure free till last follow up (12 month after operation).In $1^{\text {st }}$ six month after operation he was on Tab. phenytoin $100 \mathrm{mg}$ daily then it was stopped. His hearing was also normal at last follow up. Histopathology reported grade 1 astrocytoma.

\section{Discussion:}

The five layered cingulate gyrus sits atop the corpus callosum and can be broadly divided into two segments: the anterior cingulate (areas 24, 25, and 33) which is concerned with vocalizing and emotional and motoric functioning involving the hands, and regulating autonomic and endocrine activities; and the posterior (area 23) cingulate which is involved in visual-spatial and tactile analysis as well as motor output and memory. The posterior cingulate gyrus is richly interconnected with the superior parietal lobe (area 7) the parahippocampal (inferior) temporal and superior temporal lobe (area 22), frontal lobe, caudate, putamen, substantia nigra, pulvinar of the thalamus, and dorsal hypothalamus. ${ }^{1}$ In addition, the posterior cingulate projects to the red nucleus in the midbrain (which also receives frontal motor fibers) and to the spinal cord. Cingulate gyrus contains fasciculum cingulum that form the Papez circuit and involved in memory mechanism. ${ }^{2}$

The neural network associated with idiopathic hyperacusis is still not well known. Hwang JH et al ${ }^{3}$ studied the brain activation of 3 middle-aged patients with mild to moderate hyperacusis by functional magnetic resonance imaging while they were listening to white noise binaurally. In addition to the temporal lobes, in all patients, sound elicited activation in the frontal lobes and occipital lobes. The parahippocampus was activated in 2 of 3 patients. Furthermore, the precentral and postcentral gyri, superior and inferior parietal lobules, thalamus, midbrain, claustrum, insula, posterior cingulate gyrus, and orbital and rectal gyrus were also activated in one patient. The neural network associated with idiopathic hyperacusis might be associated with the frontal lobes and parahippocampus. ${ }^{3}$ fMRI of brain showed threat-related words compared to neutral words activated left posterior cingulate gyrus in eighty percent person with activation most prominent in the retrosplenial region. ${ }^{4}$ Epilepsy surgery for lesions involving the cingulate gyrus represents a small fraction of all epilepsy surgery cases, with good seizure outcome and low rates of post-operative permanent deficits. Post operative complications includes temporary postoperative supplementary motor area (SMA) syndrome, mild hand or leg paresis, respectively. Postoperatively, $62 \%$ of patients were seizure-free and $76 \%$ had a satisfactory seizure outcome.$^{5}$ Assessment of morbidity following resection of cingulate gyrus gliomas bt Tate MC et $\mathrm{al}^{6}$ demonstrated that $29 \%$ of patients experienced a new or worsened neurological deficit immediately after surgery. The most common deficits were supplementary motor area (SMA) syndrome $(20 \%)$, weakness $(6 \%)$, and sensory changes $(2 \%)$. SMA syndrome than patients with anerior cingulate gyrus lesion. Only $4 \%$ of patients had a persistent neurological deficit at 6 months postoperatively. A similar morbidity profile was observed in the subset analysis of patients with tumors confined to the cingulate gyrus, with no additional morbidity related to known cingulate-specific functions. Surgical morbidity is primarily a function of 
surgical trajectory rather than the particular cingulate region resected. ${ }^{6}$ von Lehe $\mathrm{M}$, et $\mathrm{al}^{7}$ studied a series of glioma in cingulated gyrus where $18 \%$ of the tumor was located in the posterior (parietal) part of the cingulate gyrus, and in $82 \%$ the tumor was in the anterior (frontal) part. In $26 \%$ cases the glioma was solely located in the cingulate gyrus, and in $74 \%$ the tumor extended to the supracingular frontal/parietal cortex. $61 \%$ cases had seizures as the presenting symptom, $24 \%$ suffered from a hemiparesis/hemihypesthesia, and $12 \%$ had aphasic symptoms. Postoperatively, $34 \%$ cases suffered from a transient supplementary motor area syndrome, all of whom had tumors in the anterior cingulate gyrus. In the early postoperative period (30 days) a new deficit occurred in $13 \%$ cases (mild motor deficits or aphasic symptoms). One patient had a major bleeding episode 2 days after surgery and was in a persistent vegetative state. $^{7}$

Common causes of hyperacusis are head injury, ear damage (i.e from barotrauma, sounds, toxins or medication), Middle ear pathology (i.e.infection, tumor $\&$ trauma and surgery), Lyme disease, air bag deployment,viral infections involving the inner ear or facial nerve (Bell's palsy), temporomandibular joint (TMJ) syndrome, William syndrome, multiple sclerosis, irritating lesion in hearing pathway. facial nerve palsy above the geniculate ganglion of various etiologies. Very rarely it can be occurred toxins and myopathy involving stapedius muscle. ${ }^{8}$ Per operative or post operative drugs that the patient received had no report to cause hyperacusis. In this case the cause of hyperacusis was not understood by our acquired knowledge. We think, in the cingulate gyrus of the patient there might be a hearing augmentation area in the posterior cingulate gyrus connected to hearing cortex or hearing pathway that was stimulated by the surgery. If such an area was present, is it present in all human? Whether is it dormant or active? If dormant when does it become active? What are the connecting tracts? Can it be applied for the treatment of deafness? Till today we do not know the answers. In future we may get the answer.

\section{Reference:}

1. Deveinsky O.,Morrell MJ.,Vogt BA. Contributions of anterior cingulate cortex to behaviour. Brain 1995; 118:279-306

2. Chowdhury FH \& Khan AH. Papez Circuit: An Anatomical Study by Cadaveric Dissection. Pan Arab Journal of Neurosurgery 2010;14(2):75-80

3. Hwang JH., Chou PH., Wu CW., Chen JH., Liu TC. Brain activation in patients with idiopathic hyperacusis. American Journal of Otolaryngology - Head and Neck Medicine and Surgery2009;30, 432-434

4. Maddock RJ, Buonocore MH. Activation of left posterior cingulate gyrus by the auditory presentation of threatrelated words: an fMRI study. Psychiatry Res. 1997 Aug $8 ; 75(1): 1-14$

5. von Lehe M, Wagner J, Wellmer J, Clusmann H, Kral T. Epilepsy surgery of the cingulate gyrus and the frontomesial cortex. Neurosurgery. 2011 Sep 23. [Epub ahead of print]

6. Tate MC, Kim CY, Chang EF, Polley MY, Berger MS. Assessment of morbidity following resection of cingulate gyrus gliomas. Clinical article. J Neurosurg. 2011 Mar;114(3):640-7. Epub 2010 Oct 8.

7. von Lehe M, Schramm J.Gliomas of the cingulate gyrus: surgical management and functional outcome. Neurosurg Focus. 2009 Aug;27(2):E9

8. Baguley DM. Hyperacusis. J R Soc Med.2003; 96(12): 582-585 